\title{
Human Wharton's jelly stem cells inhibit endometriosis through apoptosis induction
}

\author{
Saba Hajazimian¹, Masoud Maleki ${ }^{1}$ Shahla Danaei Mehrabad² and Alireza Isazadeh³ \\ ${ }^{1}$ Department of Genetics, Tabriz Branch, Islamic Azad University, Tabriz, Iran, ${ }^{2}$ Gynecology Department, Eastern \\ Azerbaijan ACECR ART Center, Eastern Azerbaijan Branch of ACECR, Tabriz, Iran and I Immunology Research \\ Center, Tabriz University of Medical Sciences, Tabriz, Iran
}

Correspondence should be addressed to M Maleki; Email: maleki.masoud@gmail.com

\begin{abstract}
Endometriosis is a relatively benign disease characterized by endometrial tumors and uterus stroma. Apoptosis suppression is one of the most important pathological processes of endometriosis. Recently, several studies reported that human Wharton's jelly stem cells (hWJSCs) can inhibit growth and proliferation of various cancer cells through induction of apoptosis. Therefore, the aim of the present study was to investigate the effects of hWJSCs conditioned medium (hWJSC-CM) and cell-free lysate (hWJSC-CL) on endometriosis cells in vitro. In the present study, effects of different concentrations of hWJSC-CM and hWJSC-CL on viability and proliferation, morphological alterations, colony formation, migration, invasion, and apoptosis of endometriosis cells were evaluated. Our results showed that hWJSC-CM and hWJSC-CL decrease viability and proliferation, colony formation, migration, and invasion, as well as increase morphological alterations and apoptosis of endometriosis cells, in a concentration- and time-dependent manner. Decreased migration and invasion of treated endometriosis cells with hWJSC-CM and hWJSC-CL may be due to decrease of MMP-2 and MMP-9 gene expression. Moreover, induction of apoptosis in treated endometriosis cells can be due to regulation of apoptosisrelated genes expression, including BAX,BCL-2, SMAC, and SURVIVIN. The results of the present study suggest that hWJSC-CM and hWJSC-CL can inhibit endometriosis cells at a mild-to-moderate level through various physiological mechanisms. However, further studies on animal models are necessary to achieve more accurate results.

Reproduction (2020) 159 549-558
\end{abstract}

\section{Introduction}

Endometriosis, a chronic benign disease, is defined by stroma tissue growths and endometrial-like gland outside the uterine cavity. Endometriosis occurs in more than $10 \%$ of women at reproductive age and is one of the main cause of dysmenorrhea and infertility (Guo 2015). Despite extensive studies, pathogenesis of this disease remains unclear, and available treatment approaches which include surgical treatment and pharmacological therapy remain a controversy. Inhibition of estrogen production by pharmacological therapy prevents disease progression. However, estrogen decrease may have associated with various complication effects (Laschke \& Menger 2016). Elimination of endometriotic lesions by surgical treatment may restore fertility and resolve symptoms. However, surgical treatment is associated with numerous complications effects (Oliveira et al. 2016). Therefore, development of an effective therapeutic approach remains a primary concern (Guo et al. 2015).

Cell therapy is an important and applicable approach in treatment of various cancers. Human Wharton's jelly stem cells (hWJSCs) isolated from human umbilical cord (which was usually discarded after delivery) are a unique source of mesenchymal stem cells (MSCs) with significant clinical utility (Fong et al. 2011). The hWJSCs are also multipotent with high proliferative potential and long telomeres, which can present embryonic and mesenchymal stem cell characteristics (Fong et al. 2010). In contrast to other MSCs, the hWJSCs do not cause tumor formation in mice with suppressed immune systems (Gauthaman et al. 2012). Moreover, the hWJSCs can be harvested painlessly in a large population from discarded umbilical cords, and they multiply rapidly in vitro and maintain their stemness characteristics for many passages in serial culture (Fong et al. 2010, Gauthaman et al. 2012).

Recently, limited studies have reported antiproliferative and inhibitory effects of hWJSCs on various human cancer cells (Ganta et al. 2009, Ahn et al. 2015). Moreover, the evidence has shown that the hWJSCs suppressed proliferation of various cancer cells through the induction of apoptosis (Hendijani \& Javanmard 2015). However, underlying mechanisms of the hWJSCs antitumor activity have not been identified. Moreover, the effect of these stem cells has not been evaluated on endometriosis. 
Therefore, the aim of the present study was to investigate inhibitory effects of hWJSCs conditioned medium (hWJSC-CM) and hWJSCs cell-free lysate (hWJSC-CL) on endometriosis cells and elucidate the underlying mechanism of action.

\section{Materials and methods}

\section{Human umbilical cords collection}

Human umbilical cords were collected from ten healthy women undergoing full-term delivery at the Iran-Tabriz International Shahryar Hospital, during June to July 2018. The collected umbilical cords were transferred to Biotechnology Research Center of Tabriz Islamic Azad University in sterile Hanks balanced salt solution (HBSS) containing $1 \%$ penicillin-streptomycin (5000 units $/ \mathrm{mL}-5000 \mathrm{mg} / \mathrm{mL}$ ) antibiotics at $4{ }^{\circ} \mathrm{C}$. All participants were informed about the study and signed a consent form in accordance with the ethical standards of the 1964 Helsinki declaration and its later amendments or comparable ethical standards. Islamic Azad University, Tabriz Branch Ethics Committee, specifically approved this study.
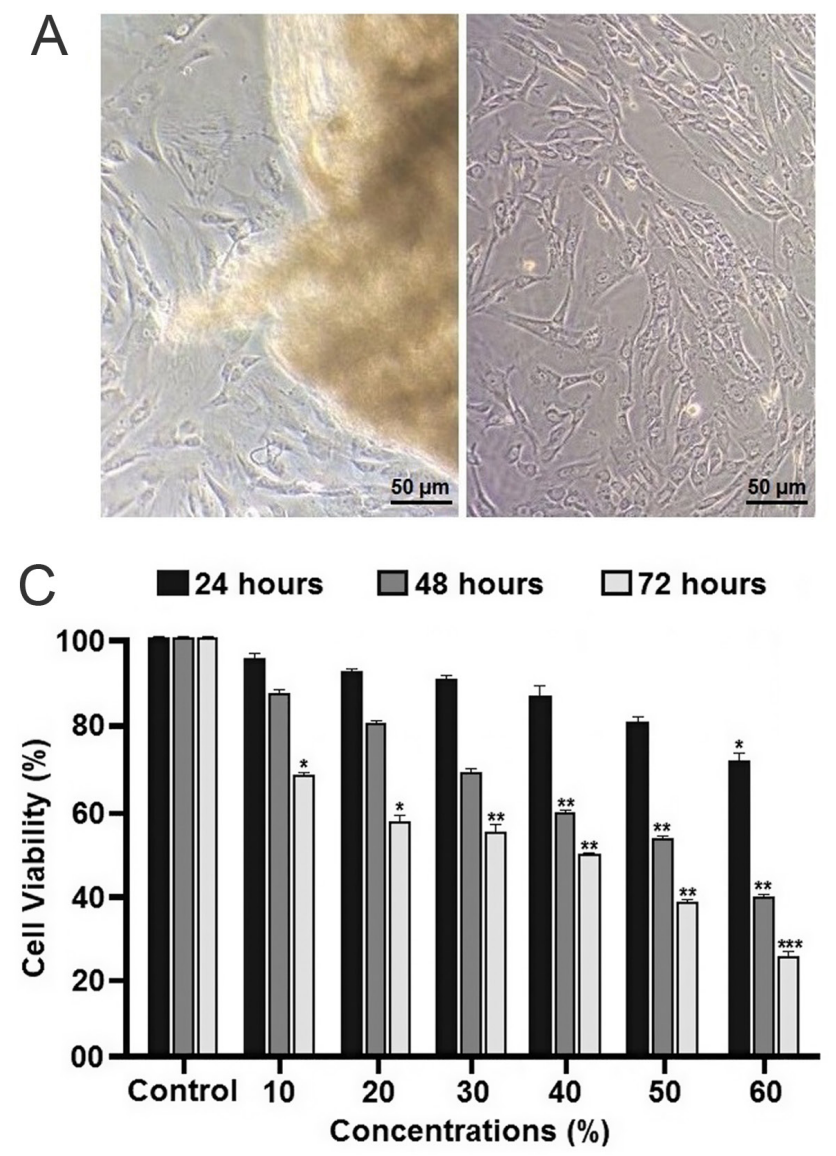

\section{Primary culture and isolation of hWJSCs}

Human umbilical cords were cut into smaller pieces and opened longitudinally. Wharton's jelly tissues were separated from umbilical cords and exposed to an enzyme cocktail, including hyaluronidase (100 IU), collagenase type-IV (2 mg/ $\mathrm{mL})$, and collagenase type-I $(2 \mathrm{mg} / \mathrm{mL})$. The matrix contents were gently scraped and then centrifuged in standard condition for cell culture (250 rpm for $10 \mathrm{~min}$ ). The cell pellet was resuspended in Dulbecco's Modified Eagle Medium (DMEM) culture medium supplemented with $10 \%$ fetal bovine serum (FBS) and $1 \%$ penicillin-streptomycin (5000 units/mL-5000 $\mathrm{mg} / \mathrm{mL}$ ) antibiotics, seeded in a T25 tissue culture flask, and incubated in the standard conditions of cell culture $\left(5 \% \mathrm{CO}_{2}\right.$ and $95 \%$ air at $37^{\circ} \mathrm{C}$ ). The culture medium was changed every $72 \mathrm{~h}$ and passaged in approximately $70 \%$ cells confluent. Finally, the biological properties of the derived hWJSCs were evaluated (Fig. 1A).

\section{Preparation of hWJSCs conditioned medium}

The derived hWJSCs were incubated in standard condition until approximately $70 \%$ cells confluent. The culture medium was changed with fresh medium (without antibiotic and FBS)
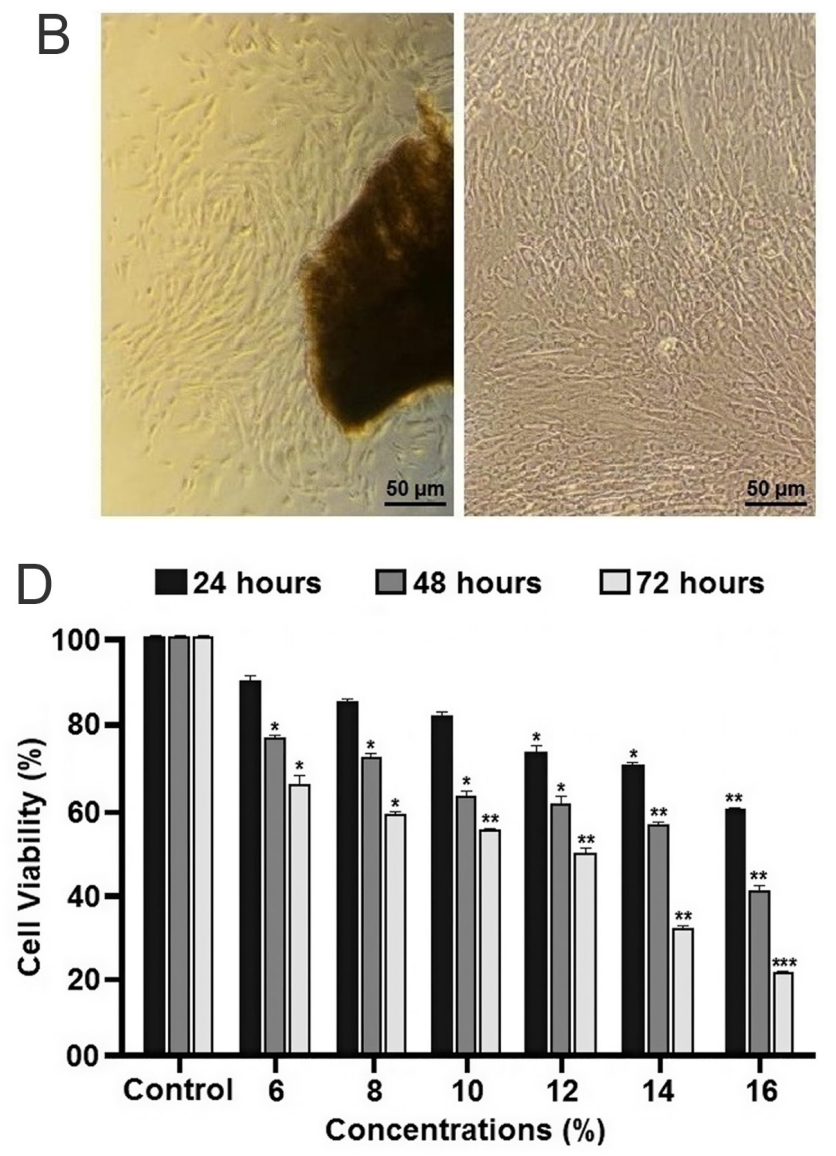

Figure 1 The inverted phase-contrast images from primary cultured hWJSCs (A) and primary cultured endometriosis cells (B) in early and late passages, which shows the short fibroblasts characteristic in early passages and long fibroblasts characteristic in later passages. The endometriosis cell viability treated with hWJSC-CM (C) and hWJSC-CL (D) at 24-72 h. The results of the three experiments are presented as mean and S.D. values $(* P<0.01 ; * * P<0.01 ; * * * P<0.001)$. 
and incubated in standard condition for $72 \mathrm{~h}$. The hWJSCs conditioned medium (hWJSC-CM) was collected, sterilized using a $0.22 \mu \mathrm{m}$ syringe filter PVDF (Sigma-Aldrich) and stored at $-20^{\circ} \mathrm{C}$.

\section{Preparation of hWJSC cell lysate}

The derived hWJSCs were incubated in standard condition until approximately $70 \%$ cells confluent. The cultured hWJSCs were washed using phosphate buffered saline (PBS), trypsinized, centrifuged, and the cells pellet exposed to cell lysis buffer (150 mM NaCl, 1.0\% Nonyl Phenoxypolyethoxylethanol-NP40, $50 \mathrm{mM}$ Tris-Cl, $1.0 \%$ sodium deoxycholate, $0.1 \%$ SDS) containing protease inhibitor cocktail. The cells suspension was centrifuged and the supernatant was collected as hWJSCs cell lysate (hWJSC-CL) and stored at $-20^{\circ} \mathrm{C}$.

\section{Human endometriosis tissue collection}

Endometriosis tissue collection was performed on three Iranian patients with deep ovarian endometrioma referred to the Iran-Tabriz International Shahryar Hospital, during June to October 2018. The studied patients were not exposed with any hormonal therapy. Moreover, all the endometriosis tissues were obtained in early secretory phase to maintain high cell proliferation capacity. The collected endometriosis tissues were transferred to Biotechnology Research Center of Tabriz Islamic Azad University in sterile HBSS containing $1 \%$ penicillinstreptomycin (5000 units $/ \mathrm{mL}-5000 \mathrm{mg} / \mathrm{mL}$ ) antibiotics at $4{ }^{\circ} \mathrm{C}$. All participants were informed about the study and signed a consent form in accordance with the ethical standards of the 1964 Helsinki Declaration and its later amendments or comparable ethical standards. Islamic Azad University, Tabriz Branch Ethics Committee, specifically approved this study.

\section{Primary culture and isolation of endometriosis cells}

Endometriosis tissues were cut into smaller pieces and washed using PBS at room temperature. The smaller endometriosis tissues were exposed to Trypsin-EDTA $(0.5 \%)$ for $10 \mathrm{~min}$ at $37^{\circ} \mathrm{C}$, suspended in RPMI culture medium supplemented with $10 \%$ FBS and $1 \%$ penicillin-streptomycin (5000 units/ $\mathrm{mL}-5000 \mathrm{mg} / \mathrm{mL}$ ) antibiotics, and then centrifuged. The cell pellet was resuspended in complete RPMI culture medium, seeded in a T25 tissue culture flask, and incubated in standard conditions. The culture medium was changed every $72 \mathrm{~h}$ and passaged in approximately $70 \%$ cells confluent. Finally, biological properties of the derived endometriosis cells were evaluated (Fig. 1B).

\section{Endometriosis cell viability assay}

The derived endometriosis cells were plated $\left(1.5 \times 10^{4}\right.$ cells/ well) in a 96-well tissue culture plate and incubated in standard condition for $24 \mathrm{~h}$. The old culture medium was substituted with fresh complete medium containing different concentrations of hWJSC-CM $(10,20,30,40,50$, and 60\%) and hWJSC-CL (6, $8,10,12,14$, and $16 \%$ ) and incubated in standard condition for 24-72 h. Moreover, cell lysis buffer with protease inhibitor cocktail were considered as negative controls. Endometriosis cell viability was evaluated using Tetrazolium Micro-culture (MTT) method. The old culture medium was substituted with $200 \mu \mathrm{L}$ complete medium containing $50 \mu \mathrm{L}$ MTT agent solution in PBS $(2 \mathrm{mg} / \mathrm{mL})$ and incubated in standard condition for $4 \mathrm{~h}$. The old culture medium was removed and $50 \mu \mathrm{L}$ dimethyl sulfoxide (DMSO) was added and incubated in standard condition for $30 \mathrm{~min}$. Optical density (OD) was measured using a microplate ELISA reader instrument at $570 \mathrm{~nm}$ wavelength. Finally, viability percentage of treated endometriosis cells were measured using the following formula: ((treated sample OD / untreated sample OD) $\times 100)$.

\section{Endometriosis cell morphology assay}

The derived endometriosis cells were plated $\left(1.5 \times 10^{5}\right.$ cells/ well) in a 6-well tissue culture plate and incubated in standard condition for $24 \mathrm{~h}$. The old culture medium was substituted with complete medium containing different concentrations of hWJSC-CM (30, 40, and 50\%) and hWJSC-CL (10, 12, and $14 \%$ ) and incubated in standard condition for 24-72 h. Any visible morphological alterations were monitored every $24 \mathrm{~h}$ using an inverted phase-contrast microscopy.

\section{Endometriosis cell transwell migration assays}

The derived endometriosis cells were plated $\left(1 \times 10^{5}\right.$ cells/ well) in the upper chamber of a transwell chamber $(8 \mu \mathrm{m}$ pore size) in the serum-free RPMI medium containing different concentrations of hWJSC-CM (30, 40, and 50\%) and hWJSC-CL (10, 12, and 14\%). Moreover, the RPMI medium supplemented with $10 \%$ FBS was added to the bottom chamber and incubated in standard condition for $24 \mathrm{~h}$. The unmigrated cells on the upper chamber were removed. Also, the migrated cells on the bottom chamber were fixed using paraformaldehyde (4\%) and stained using crystal violet $(0.1 \%)$ for $24 \mathrm{~h}$. The migrated cells were counted and averaged in the five random areas of the transwell membrane using an inverted phase-contrast microscopy.

\section{Endometriosis cell wound-healing assay}

The derived endometriosis cells were plated $\left(1.5 \times 10^{5}\right.$ cells/ well) in a 6-well tissue culture plate and incubated in standard condition for $24 \mathrm{~h}$. The center of each wells with monolayer cells were scratched using a pipette tips and washed with PBS to remove detached cells. The old culture medium was substituted with complete medium containing hWJSC-CM $(40 \%)$ and hWJSC-CL $(12 \%)$ and incubated in standard condition for $30 \mathrm{~h}$. The migration of endometriosis cells in the denuded area were monitored using an inverted phasecontrast microscopy at $0,10,20$, and $30 \mathrm{~h}$.

\section{Endometriosis cell colony formation assay}

The derived endometriosis cells were plated $\left(1.5 \times 10^{5}\right.$ cells/ well) in a 6 -well tissue culture plate and incubated in standard condition for $24 \mathrm{~h}$. The old culture medium was substituted with complete medium containing different concentrations 
of hWJSC-CM $(10,20,30,40$, and 50\%) and hWJSC-CL $(8,10,12,14$, and $16 \%)$ and incubated in standard condition. The culture medium was changed after $72 \mathrm{~h}$ and incubated in standard conditions for 2 weeks. The endometriosis cell colonies were fixed using paraformaldehyde (4\%) and stained using crystal violet $(0.1 \%)$ for $24 \mathrm{~h}$. The colony formation was counted and averaged using an inverted phase-contrast microscopy.

\section{Endometriosis cell apoptosis assay}

The derived endometriosis cells were plated $\left(1.5 \times 10^{5}\right.$ cells/ well) in a 6-well tissue culture plate and incubated in standard condition for $24 \mathrm{~h}$. The old culture medium was substituted with complete medium containing different concentrations of hWJSC-CM (20 and 40\%) and hWJSC-CL (8 and 12\%) and incubated in standard condition for $24 \mathrm{~h}$. The cells were washed with ice-cold PBS, trypsinized, centrifuged, and resuspended in binding buffer containing propidium iodideand annexin V. The cells suspension $(100 \mu \mathrm{L})$ was incubated in standard condition, darkness, and room temperature with $\mathrm{PI}$ $(10 \mathrm{~mL})$, Annexin V-Fluorescein Isothiocyanate (FITC) $(5 \mathrm{~mL})$, and binding buffer $(400 \mu \mathrm{L})$ for $15 \mathrm{~min}$. Finally, quantitative apoptosis was determined using FITC/Propidium lodide (PI) by flowcytometry instrument.

\section{Genes expression analysis}

The derived endometriosis cells were plated $\left(1.5 \times 10^{5}\right.$ cells/ well) in a 6-well tissue culture plate and incubated in standard condition for $24 \mathrm{~h}$. The old culture medium was substituted with complete medium containing different concentrations of hWJSC-CM (30 and 40\%) and hWJSC-CL (8 and 12\%) and incubated in standard condition for $72 \mathrm{~h}$. The total RNA extraction was performed using Trizol reagent, according to the manufacturer's instructions. The synthesizes of cDNA was performed using RT and random hexamers. The quality and quantity of extracted RNA were investigated using electrophoresis on $1 \%$ agarose gel and NanoDrop instrument. The mRNA expression of SMAC, SURVIVIN, BAX, BCL-2, $M M P-2$, and MMP-9 genes were evaluated using Real-Time PCR. The used primers sequences are presented in Table 1. The Real-Time PCR was performed using $5 \mu \mathrm{L}$ Master Mix, $1 \mu \mathrm{g}$ CDNA, $0.5 \mu \mathrm{L}$ forward primer, and $0.5 \mu \mathrm{L}$ reverse primer, in $10 \mu \mathrm{L}$ total volume, as follows: one cycle for initial denaturation in $94^{\circ} \mathrm{C}$ for $1 \mathrm{~min}, 40$ cycles for denaturation in $94^{\circ} \mathrm{C}$ for $10 \mathrm{~s}, 40$ cycles for annealing in $59^{\circ} \mathrm{C}$ for $30 \mathrm{~s}$, and 40 cycles for extension in $72^{\circ} \mathrm{C}$ for $20 \mathrm{~s}$. Also, $\beta$-actin gene was considered as endogenous control. The data were analyzed by comparative $2^{-\Delta \Delta \mathrm{Ct}}$ threshold cycle.

\section{Statistical analysis}

All mentioned experiments were repeated for three times. The obtained data from three independent experiments were presented as mean \pm S.D. Statistical analysis including one-way ANOVA, Tukey (post hoc), and Student's $t$ test was performed using the Graph Pad Prism software. A $P$-value of $<0.05$ was considered statically significant.
Table 1 The sequences and characteristics of primers used for detection of genes expression.

\begin{tabular}{|c|c|c|c|}
\hline Genes & Primer sequence & $T_{m}$ & $\begin{array}{c}\text { Produc } \\
\text { size }\end{array}$ \\
\hline \multirow[t]{3}{*}{$B A X$} & & & $155 \mathrm{bp}$ \\
\hline & F-CCCGAGAGGTCTTTTTCCGAG & $60^{\circ} \mathrm{C}$ & \\
\hline & R-CCAGCCCATGATGGTTCTGAT & $60^{\circ} \mathrm{C}$ & \\
\hline \multirow[t]{3}{*}{$B C L-2$} & & & $223 \mathrm{bp}$ \\
\hline & F-GATGGGATCGTTGCCTTATG & $56^{\circ} \mathrm{C}$ & \\
\hline & R-GCGGAACACTTGATTCTGG & $56^{\circ} \mathrm{C}$ & \\
\hline \multirow[t]{3}{*}{ SMAC } & & & $196 \mathrm{bp}$ \\
\hline & F-CAGAGGAGGAAGATGAAGTGTG & $55^{\circ} \mathrm{C}$ & \\
\hline & R-GCGGTTATAGAGGCCTGATCTG & $56^{\circ} \mathrm{C}$ & \\
\hline \multirow[t]{3}{*}{ SURVIVIN } & & & $172 \mathrm{bp}$ \\
\hline & F-СССТTTCTCAAGGACCACCG & $52^{\circ} \mathrm{C}$ & \\
\hline & R-GTTCCTCTATGGGGTCGTCA & $51^{\circ} \mathrm{C}$ & \\
\hline \multirow[t]{3}{*}{$M M P-2$} & & & $177 \mathrm{bp}$ \\
\hline & F-CACATAGTGATGGTTCCССTGTT & $60^{\circ} \mathrm{C}$ & \\
\hline & R-CGGCCACTCAGTAGGTGTCTTT & $62^{\circ} \mathrm{C}$ & \\
\hline \multirow[t]{3}{*}{ MMP-9 } & & & $187 \mathrm{bp}$ \\
\hline & F-ATTTCTGCCAGGACCGCTTCTAC & $62^{\circ} \mathrm{C}$ & \\
\hline & R-ATCCGGCAAACTGGCTCCTTC & $63^{\circ} \mathrm{C}$ & \\
\hline \multirow[t]{3}{*}{$A C T B$} & & & $186 \mathrm{bp}$ \\
\hline & F-AGAGCTACGAGCTGCCTGAC & $61^{\circ} \mathrm{C}$ & \\
\hline & R-AGCACTGTGTTGGCGTACAG & $59^{\circ} \mathrm{C}$ & \\
\hline
\end{tabular}

\section{Results}

\section{Endometriosis cell viability}

Endometriosis cell viability decreased in a time and concentration-dependent manner following treatment with different concentrations of hWJSC-CM $(10,20$, $30,40,50$, and $60 \%$ ) and hWJSC-CL $(6,8,10,12$, 14 , and $16 \%$ ) at 24,48 , and 72 h. Viability of the treated endometriosis cells significantly decreased in high concentrations of hWJSC-CM and hWJSC-CL, as compared with untreated controls (Fig. 1C and D). However, anti-proliferation effects of hWJSC-CL was significantly more than hWJSC-CM in the same concentrations. The inhibition concentration $\left(\mathrm{IC}_{50}\right)$ of hWJSC-CM and hWJSC-CL on endometriosis cells after $72 \mathrm{~h}$ were 40 and $12 \%$, respectively.

\section{Endometriosis cell morphology}

Endometriosis cells showed various morphological alterations following treatment with different concentrations of hWJSC-CM $(30,40$, and 50\%) and hWJSC-CL $(10,12$, and $14 \%)$ at 24,48 , and $72 \mathrm{~h}$. The observed morphological alterations include cell shrinkage, decrease in the size of cells, condensed or fragmented nuclei, membrane damages, chromatin condensation, and formation of apoptotic bodies. These cellular alterations can cause cell death following treatment with hWJSC-CL (Fig. 2A) and hWJSC-CM (Fig. $2 \mathrm{~B})$. The cellular alterations were concentration- and time-dependent and were more evident with higher concentrations. 

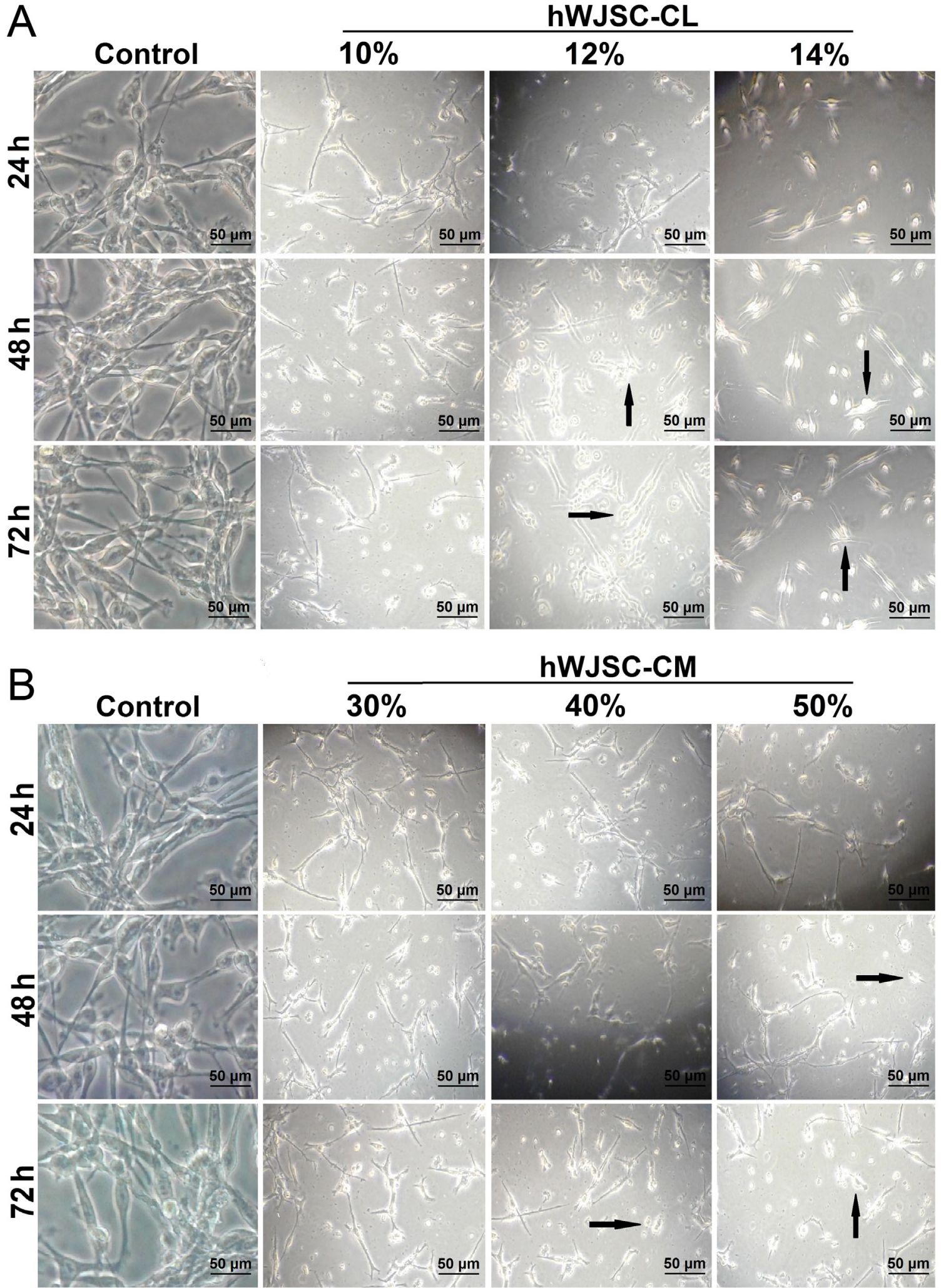

Figure 2 The inverted phase-contrast images from morphological alterations of endometriosis cells treated with hWJSC-CL (A) and hWJSC-CM (B). The morphological alterations were more evident in endometriosis cells treated with high concentrations of hWJSC-CM or hWJSC-CL and long time. The observed morphological alterations include cell shrinkage, decrease in the size of cells, condensed or fragmented nuclei, membrane damages, chromatin condensation, and formation of apoptotic bodies. 


\section{Endometriosis cell migration and invasion}

In the transwell assay, migration of endometriosis cells decreased significantly in a concentration-dependent mannerfollowing treatmentwith differentconcentrations of hWJSC-CM (30, 40, and 50\%) and hWJSC-CL (10, 12 , and $14 \%$ ), as compared with untreated controls (Fig. $3 \mathrm{~A}$ and $\mathrm{C}$ ). In the wound-healing assay, also the invasion of endometriosis cells decreased significantly in a time-dependent manner following treatment with hWJSC-CM (30\%) and hWJSC-CL (12\%) (Fig. 3B and D). To investigate whether hWJSC-CM and hWJSC-CL inhibit migration and invasion of endometriosis cells, we evaluated mRNA expression of MMP-2 and MMP9 genes. The Real-Time PCR analysis showed a lower expression of MMP-2 and MMP-9 genes in treated endometriosis cells with hWJSC-CM (30 and 40\%) and hWJSC-CL (8 and 12\%), as compared with untreated controls. The hWJSC-CM and hWJSC-CL significantly decreased mRNA expression of MMP-2 and MMP-9 genes in endometriosis cells. Our study suggested a key role of MMP-2 and MMP-9 genes in migration and invasion of endometriosis cells (Fig. 3E and F).

\section{Endometriosis cell colony formation}

The colony formation, colony diameter, and colony size of endometriosis cells were decreased significantly following treatment with different concentrations of hWJSC-CM (10, 20, 30, 40, and 50\%) and hWJSC-CL $(8,10,12,14$, and 16\%) (Fig. 4A and B). The colony formation, inhibited in a concentration-dependent manner, was more inhibited with higher concentrations of hWJSC-CM and hWJSC-CL (Fig. 4C and D).
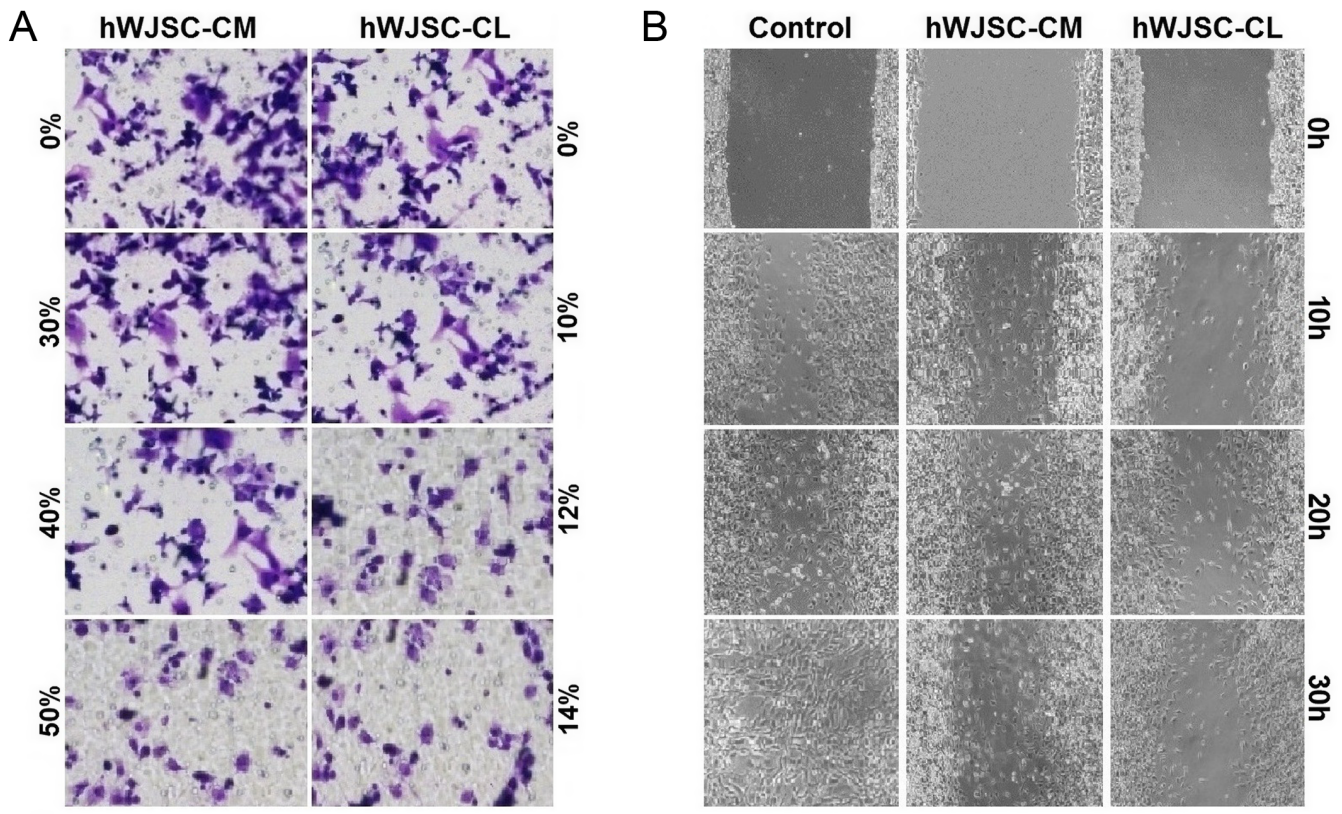

C

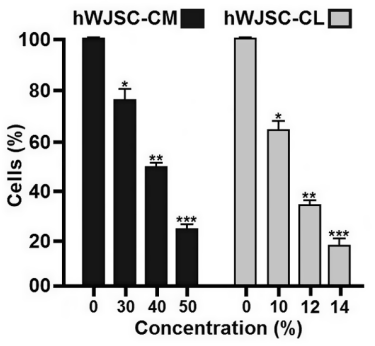

D

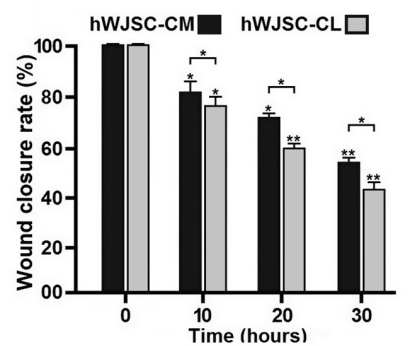

$\mathrm{E}$

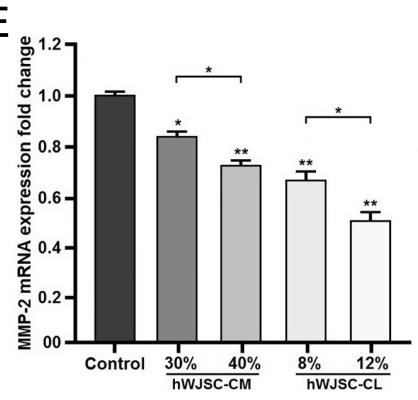

웅

$\mathrm{F}$

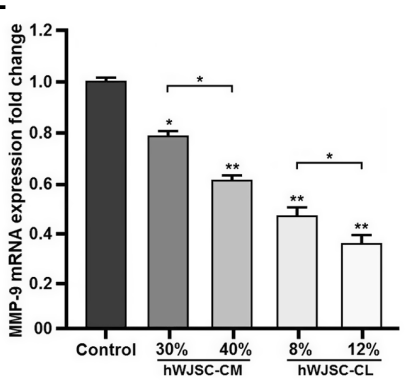

Figure 3 The inverted phase-contrast images from the effects of hWJSC-CM and hWJSC-CL on migration and invasion in endometriosis cells (A and B). The migration of endometriosis cells significantly decreased following treatment with hWJSC-CM (30, 40, and 50\%) and hWJSC-CL (10, 12 , and $14 \%$ ), assessed using transwell migration assays (C). The invasion of endometriosis cells significantly decreased following treatment with hWJSC-CM (40\%) and hWJSC-CL (12\%), assessed using scratch wound healing assay (D). The expression of MMP-2 and MMP-9 genes in endometriosis cells significantly decreased following treatment with hWJSC-CM (30,40\%) and hWJSC-CL (8 and 12\%), assessed using Real-Time PCR analysis (E, F). The results of the three experiments are presented as mean and S.D. values $\left({ }^{*} P<0.01 ;{ }^{* *} P<0.01\right)$. 


\section{Endometriosis cell apoptosis}

Apoptosis quantifying revealed that the both early and late apoptosis increased significantly in endometriosis cells treated with different concentrations of hWJSC-CM (20 and $40 \%$ ) and hWJSC-CL (8 and 12\%), as compared with untreated controls. Total apoptosis in endometriosis cells treated with hWJSC-CM and hWJSC-CL increased in a concentration-dependent manner. Induction of apoptosis by hWJSC-CL cells was significantly more than hWJSC-CM (Fig. 5A and B). Moreover, evaluation of apoptosis related genes showed that the hWJSC-CM (30 and $40 \%$ ) and hWJSC-CL (8 and 12\%) significantly increased mRNA expression of BAX and SMAC genes (Fig. 5C and D) and significantly decreased mRNA expression of BCL-2 and SURVIVIN genes (Fig. 5E and F) in endometriosis cells, as compared with untreated controls. Regulation of apoptosis related gene expression in endometriosis cells by hWJSC-CL was significantly more than hWJSC-CM.

\section{Discussion}

Endometriosis is one of the most common women diseases worldwide. In recent years, the incidence of endometriosis has increased due to various genetic and environmental factors (Tingen et al. 2018). One of the important pathological processes of endometriosis is the suppression of apoptosis (Sbracia et al. 2016). The hWJSCs derived from umbilical cord is considered as an anticancer agent against various human cancers (Liu et al. 2017, Yuan et al. 2017). The hWJSCs have many advantages compared to other currently available MSCs, such as painless harvest, as they are derived from umbilical cord which are usually discarded at birth, relatively young when derived, highly proliferative, have long telomeres, and are widely multipotent (Fong et al. 2010).

In present study, we evaluated the cell viability and proliferation, apoptosis, morphological alterations, and colony formation in hWJSC-CM and hWJSC-CL treated endometriosis cells, as compared with untreated controls. We observed that hWJSC-CM and hWJSC-CL significantly decreased the endometriosis cell viability, proliferation, and metabolic activities, as well as colony formation. Moreover, hWJSC-CM and hWJSC-CL significantly increased the morphological alterations in endometriosis cells, includes cell shrinkage, chromatin condensation, and apoptotic bodies formation, which clearly showed that the inhibition of endometriosis cells may be due to apoptosis induction. Recent studies reported many morphological alterations in various cancer cells treated with MSCs, which cause cell death (Gauthaman et al. 2012, 2013). A study by Kalamegam et al. (2018) reported that extraction of hWJSCs cause cellular damage, inhibition of cellular growth, proliferation, and induction apoptosis in ovarian cancer cells (Kalamegam et al. 2018). Another study by Han et al. (2014) also reported that mesenchymal stem cells derived from human cord cause inhibition of cellular growth and proliferation in prostate cancer cells (Han et al. 2014).

The high complications in patients with endometriosis is due to metastasis and invasion of endometriosis cells (Santamaria et al. 2012). To further identify the effects of hWJSC-CM and hWJSC-CL on the inhibition of endometriosis cells, we used the wound healing and transwell migration assay methods. Our study indicated that invasion and migration of endometriosis cells
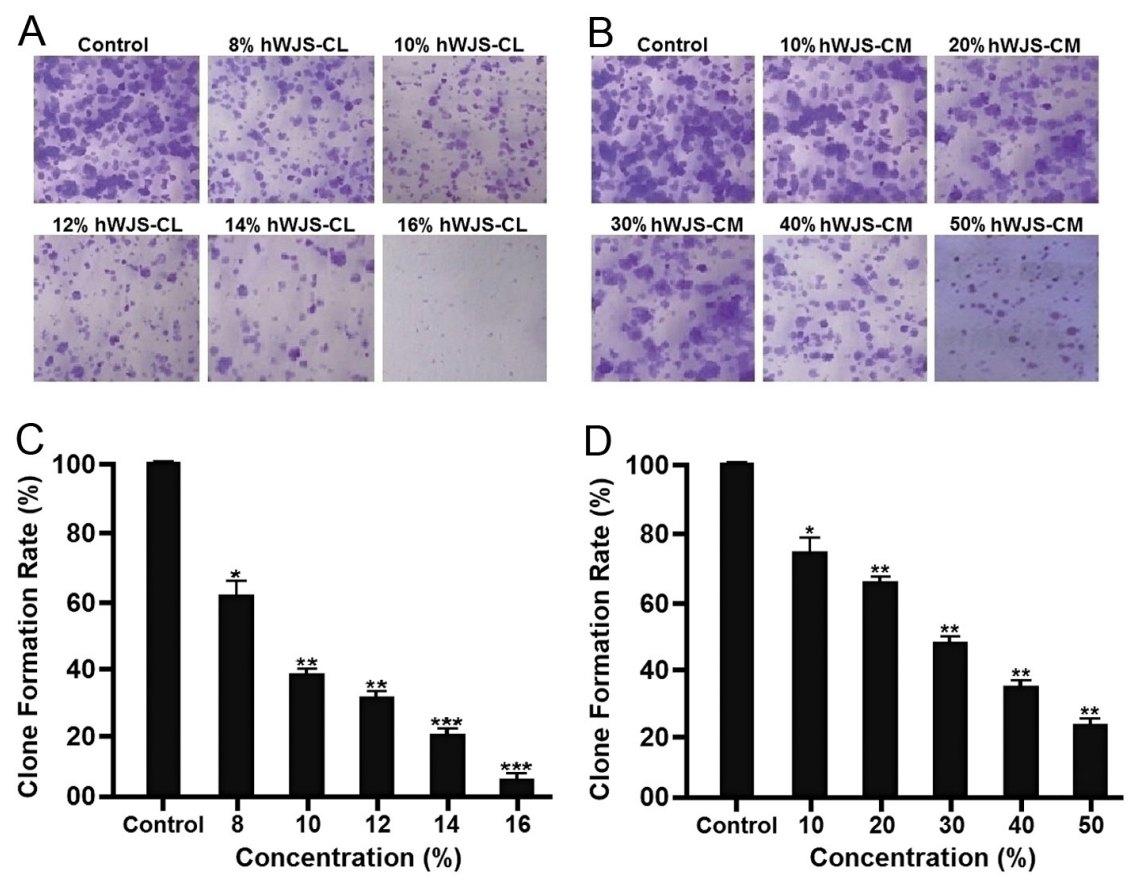

Figure 4 The inverted phase contrast images from the effects of hWJSC-CM and hWJSC-CL on the endometriosis cell colony formation (A and $\mathrm{B}$ ). The colony formation of endometriosis cells significantly decreased following treatment with hWJSC-CL $(8,10,12,14$, and $16 \%)$ and hWJSC-CM $(10,20,30,40$, and $50 \%$ ), as compared with untreated controls (C and D). The results of the three experiments are presented as mean and S.D. values $\left({ }^{*} P<0.01 ;{ }^{* *} P<0.01 ;{ }^{* * *} P<0.001\right)$. 

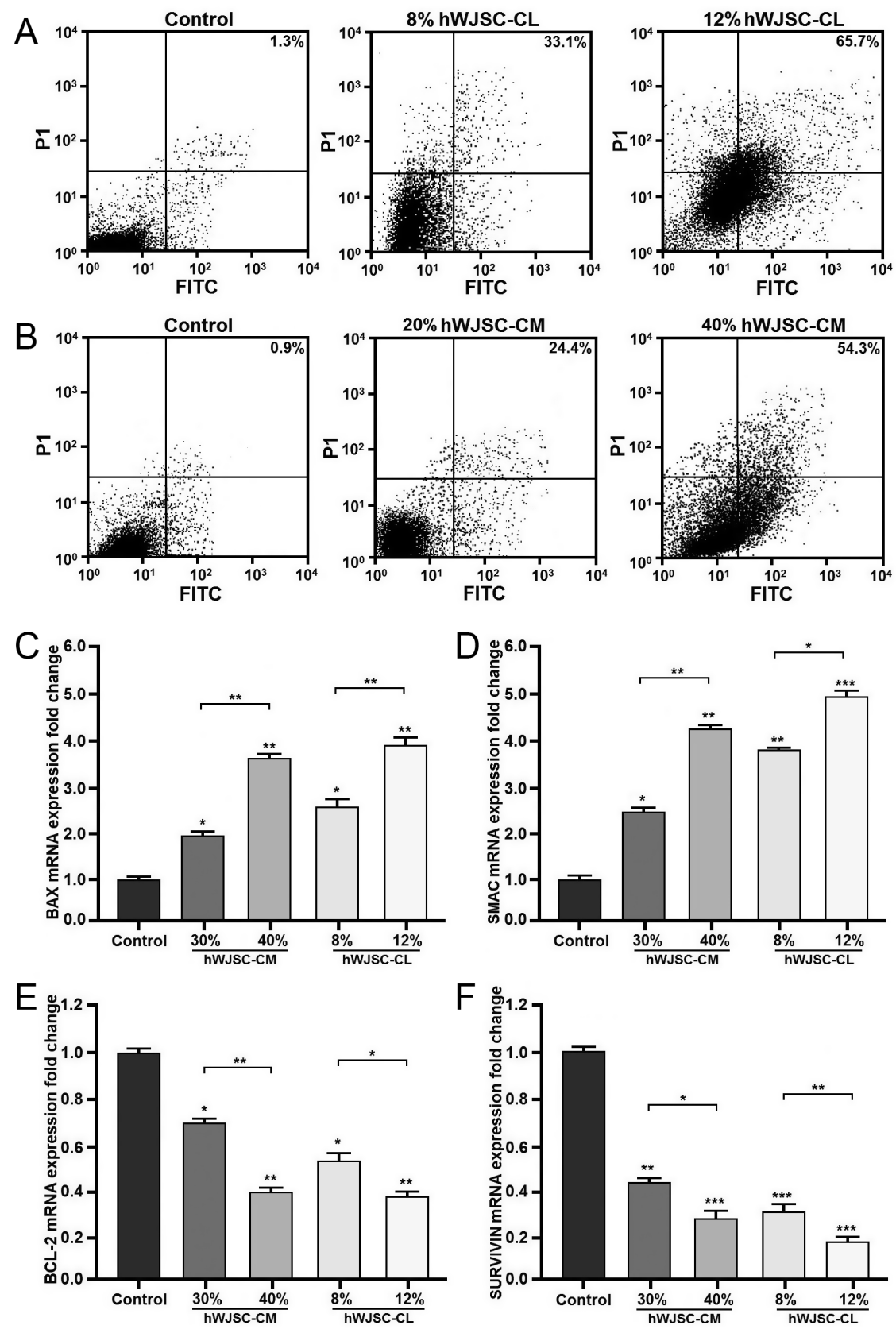

Figure 5 The effects of hWJSC-CM and hWJSC-CL on apoptosis of endometriosis cell. The apoptosis of endometriosis cells significantly increased following treatment with hWJSC-CL (8 and 12\%) and hWJSC-CM (20 and $40 \%$ ), as compared with untreated controls (A and B). The expression of BAX and $S M A C$ genes significantly increased (C and $D)$, as well as the expression of $B C L-2$ and SURVIVIN genes significantly decreased in endometriosis cells ( $E$ and $F$ ), as compared with untreated controls. The results of the three experiments are presented as mean and S.D. values $\left({ }^{*} P<0.01 ;{ }^{* *} P<0.01 ;{ }^{* * *} P<0.01\right)$.

were significantly inhibited following treatment with hWJSC-CM and hWJSC-CL. To further investigate the effects of hWJSC-CM and hWJSC-CL on the inhibition of migration and invasion of endometriosis cells, we evaluated the MMP-2 and MMP-9 genes expression in hWJSC-CM and hWJSC-CL treated endometriosis cells. Previous studies demonstrated that MMP-2 and MMP-9 played an important role in cell proliferation and also involved in invasion and metastasis of various cancer cells (Lu et al. 2012, Merdad et al. 2014). Moreover, the MMP-2 and MMP-9 genes are overexpressed in approximately all human cancers and considered as potential targets for cancer therapy (Egeblad \& Werb 2002). The obtained results indicated that hWJSC-CM and hWJSC-CL significantly inhibited the expression of MMP-2 and MMP-9 in endometriosis cells. This result indicated that hWJSC-CM and hWJSC-CL may inhibit the invasion and migration of endometriosis cells by inhibition of MMPs expression.

Induction of apoptosis is one of the most important approach for cancer cell death following treatment with various anticancer compounds. Previous studies reported that hWJSC-CM and hWJSC-CL cause apoptosis induction in various cancer cells (Gauthaman et al. 2013, Kalamegam et al. 2018). In this study, cell apoptosis was investigated by DAPI staining and Annexin $\mathrm{V}$-FITC/PI. The obtained results revealed that apoptotic rates of endometriosis cells significantly increased 
following treatment with hWJSC-CM and hWJSC-CL, proving induction of apoptosis in endometriosis cells. The intrinsic- or mitochondrial apoptosis pathway is usually regulated by interaction between various genes family members (Hockenbery et al. 1993). The high expression of $B A X$ and SMAC genes, as well as low expression of BCL-2 and SURVIVIN genes, causes activation of caspases and apoptosis onset (Huerta et al. 2007, Chelluboina et al. 2014). Our study showed that hWJSC-CM and hWJSC-CL significantly upregulated $B A X$ and SMAC gene expression, as well as downregulated $B C L-2$ and SURVIVIN gene expression in endometriosis cells. These results suggested the effects of hWJSC-CM and $\mathrm{hWJSC}-\mathrm{CL}$ on intrinsic-apoptotic pathway and induction of apoptosis in endometriosis cells.

In general, our study represents a novel therapeutic approach to treatment of patients with endometriosis. We suggested that hWJSC-CM and hWJSC-CL cause induction of intrinsic-apoptotic pathway in endometriosis cells. Therefore, hWJSCs can be used in the future to control and even treat patients with endometriosis. However, further studies are necessary to explain the exact effects of hWJSCs on physiopathology of endometriosis.

\section{Declaration of interest}

The authors declare that there is no conflict of interest that could be perceived as prejudicing the impartiality of the research reported.

\section{Funding}

This research was supported by funds from the Islamic Azad University, Tabriz Branch.

\section{Author contribution statement}

M M designed the study concept, analyzed data, and prepared the manuscript. $\mathrm{S} \mathrm{H}$ conducted experimental studies and drafted the manuscript. S D M and A I involved in drafting the manuscript and preparing the final revision of the manuscript.

\section{Acknowledgments}

This article was extracted from the MSc project of Saba Hajazimian, where M M supervised and S D M advised this project. The authors thank the whole staff of the Immunology Research Center of Tabriz University of Medical Sciences for their assistance in this research.

\section{References}

Ahn JO, Coh YR, Lee HW, Shin IS, Kang SK \& Youn HY 2015 Human adipose tissue-derived mesenchymal stem cells inhibit melanoma growth in vitro and in vivo. Anticancer Research 35 159-168.

Chelluboina B, Klopfenstein JD, Gujrati M, Rao JS \& Veeravalli KK 2014 Temporal regulation of apoptotic and anti-apoptotic molecules after middle cerebral artery occlusion followed by reperfusion. Molecular Neurobiology 49 50-65. (https://doi.org/10.1007/s12035-013-8486-7)

Egeblad M \& Werb Z 2002 New functions for the matrix metalloproteinases in cancer progression. Nature Reviews: Cancer 2 161-174. (https://doi. org/10.1038/nrc745)

Fong CY, Subramanian A, Biswas A, Gauthaman K, Srikanth P, Hande MP \& Bongso A 2010 Derivation efficiency, cell proliferation, freeze-thaw survival, stem-cell properties and differentiation of human Wharton's jelly stem cells. Reproductive Biomedicine Online 21 391-401. (https:// doi.org/10.1016/j.rbmo.2010.04.010)

Fong CY, Chak LL, Biswas A, Tan JH, Gauthaman K, Chan WK \& Bongso A 2011 Human Wharton's jelly stem cells have unique transcriptome profiles compared to human embryonic stem cells and other mesenchymal stem cells. Stem Cell Reviews and Reports 7 1-16. (https:// doi.org/10.1007/s12015-010-9166-x)

Ganta C, Chiyo D, Ayuzawa R, Rachakatla R, Pyle M, Andrews G, Weiss M, Tamura M \& Troyer D 2009 Rat umbilical cord stem cells completely abolish rat mammary carcinomas with no evidence of metastasis or recurrence 100 days post-tumor cell inoculation. Cancer Research 69 1815-1820. (https://doi.org/10.1158/0008-5472.CAN-08-2750)

Gauthaman K, Fong CY, Suganya CA, Subramanian A, Biswas A, Choolani M \& Bongso A 2012 Extra-embryonic human Wharton's jelly stem cells do not induce tumorigenesis, unlike human embryonic stem cells. Reproductive Biomedicine Online 24 235-246. (https://doi. org/10.1016/j.rbmo.2011.10.007)

Gauthaman K, Fong CY, Arularasu S, Subramanian A, Biswas A, Choolani M \& Bongso A 2013 Human Wharton's jelly stem cell conditioned medium and cell-free lysate inhibit human osteosarcoma and mammary carcinoma cell growth in vitro and in xenograft mice. Journal of Cellular Biochemistry 114 366-377. (https://doi.org/10.1002/jcb.24367)

Guo SW 2015 Endometriosis and ovarian cancer: potential benefits and harms of screening and risk-reducing surgery. Fertility and Sterility $\mathbf{1 0 4}$ 813-830. (https://doi.org/10.1016/j.fertnstert.2015.08.006)

Guo SW, Ding D, Geng JG, Wang L \& Liu X 2015 P-selectin as a potential therapeutic target for endometriosis. Fertility and Sterility 103 990-1000. e8. (https://doi.org/10.1016/j.fertnstert.2015.01.001)

Han I, Yun M, Kim EO, Kim B, Jung MH \& Kim SH 2014 Umbilical cord tissue-derived mesenchymal stem cells induce apoptosis in PC-3 prostate cancer cells through activation of JNK and downregulation of PI3K/AKT signaling. Stem Cell Research and Therapy 5 54. (https://doi. org/10.1186/scrt443)

Hendijani F \& Javanmard SH 2015 Dual protective and cytotoxic benefits of mesenchymal stem cell therapy in combination with chemotherapy/radiotherapy for cancer patients. Critical Reviews in Eukaryotic Gene Expression 25 203-207. (https://doi.org/10.1615/ CritRevEukaryotGeneExpr.2015013843)

Hockenbery DM, Oltvai ZN, Yin XM, Milliman CL \& Korsmeyer SJ 1993 $\mathrm{Bcl}-2$ functions in an antioxidant pathway to prevent apoptosis. Cell 75 241-251. (https://doi.org/10.1016/0092-8674(93)80066-n)

Huerta S, Heinzerling JH, Anguiano-Hernandez YM, Huerta-Yepez S, Lin J, Chen D, Bonavida B \& Livingston EH 2007 Modification of gene products involved in resistance to apoptosis in metastatic colon cancer cells: roles of Fas, Apaf-1, NFkB, IAPs, Smac/Diablo, and AIF. Journal of Surgical Research 142 184-194. (https://doi.org/10.1016/j. jss.2006.12.551)

Kalamegam G, Sait KHW, Ahmed F, Kadam R, Pushparaj PN, Anfinan N, Rasool M, Jamal MS, Abu-Elmagd M \& Al-Qahtani M 2018 Human Wharton's jelly stem cell (hWISC) extracts inhibit ovarian cancer cell lines OVCAR3 and SKOV3 in vitro by inducing cell cycle arrest and apoptosis. Frontiers in Oncology 8 592. (https://doi.org/10.3389/ fonc.2018.00592)

Laschke MW \& Menger MD 2016 The gut microbiota: a puppet master in the pathogenesis of endometriosis? American Journal of Obstetrics and Gynecology 215 68.e1-68.e4. (https://doi.org/10.1016/j.ajog.2016.02.036)

Liu B, Chen F, Wu Y, Wang X, Feng M, Li Z, Zhou M, Wang Y, Wu L, Liu X et al. 2017 Enhanced tumor growth inhibition by mesenchymal stem cells derived from iPSCs with targeted integration of interleukin24 into rDNA loci. Oncotarget 8 40791-40803. (https://doi.org/10.18632/oncotarget.16584)

Lu LS, Chen L, Ding WX, Li K \& Wu JJ 2012 Elevated expression of both MDR1 and MMP-2 genes in metastasized lymph node of invasive ductal breast cancer. European Review for Medical and Pharmacological Sciences 16 2037-2043. 
Merdad A, Karim S, Schulten HJ, Dallol A, Buhmeida A, Al-Thubaity F, Gari MA, Chaudhary AG, Abuzenadah AM \& Al-Qahtani MH 2014 Expression of matrix metalloproteinases (MMPs) in primary human breast cancer: MMP-9 as a potential biomarker for cancer invasion and metastasis. Anticancer Research 34 1355-1366.

Oliveira MA, Pereira TR, Gilbert A, Tulandi T, de Oliveira HC \& De Wilde RL 2016 Bowel complications in endometriosis surgery. Best Practice and Research: Clinical Obstetrics and Gynaecology 35 51-62. (https://doi.org/10.1016/j.bpobgyn.2015.11.002)

Santamaria X, Massasa EE \& Taylor HS 2012 Migration of cells from experimental endometriosis to the uterine endometrium. Endocrinology 153 5566-5574. (https://doi.org/10.1210/en.2012-1202)

Sbracia M, Valeri C, Antonini G, Biagiotti G, Pacchiarotti A \& Pacchiarotti A 2016 Fas and Fas-ligand in eutopic and ectopic endometrium of women with endometriosis: the possible immune privilege of ectopic endometrium. Reproductive Sciences 23 81-86. (https://doi.org/10.1177/1933719115594019)
Tingen CM, Mazloomdoost D \& Halvorson LM 2018 Gynecologic health and disease research at the Eunice Kennedy Shriver National Institute of Child Health and Human Development: a scientific vision. Obstetrics and Gynecology 132 987-998. (https://doi.org/10.1097/ AOG.0000000000002877)

Yuan Z, Kolluri KK, Gowers KH \& Janes SM 2017 TRAIL delivery by MSCderived extracellular vesicles is an effective anticancer therapy. Journal of Extracellular Vesicles 6 1265291. (https://doi.org/10.1080/20013078. 2017.1265291)

Date received 11 December 2019

First decision 15 January 2020

Revised manuscript received 12 February 2020

Accepted 14 February 2020 